\title{
Due vie per riconciliare il passato delle nazioni? \\ Dalle Commissioni storico culturali italo-slovena e italo-croata alle giornate memoriali
}

\author{
Raoul Pupo
}

\section{L'origine delle Commissioni storiche}

Tutto è cominciato con le "foibe", intendendo con tale termine le stragi di italiani avvenute nella Venezia Giulia nell'autunno del 1943 e nella primavera del $1945^{1}$. Si è trattato di un evento certo assai drammatico, ma limitato nelle dimensioni - alcune migliaia di vittime — rispetto a episodi analoghi avvenuti nel medesimo torno di tempo in luoghi non lontani, come pure circoscritto negli effetti, posto che non influì in maniera significativa né sugli assetti etnici della regione né sull'appartenenza statuale dei territori alto-adriatici annessi all'Italia dopo la prima guerra mondiale e trasferiti quasi completamente alla Jugoslavia dopo la seconda. Tuttavia, quella vicenda ha assunto un ruolo assolutamente esorbitante nella memoria italiana e, conseguentemente, nelle reazioni di parte slovena e croata, tanto da divenire il simbolo contrastato della complessa storia di violenze che si snodò fra il tempo di guerra e la metà degli anni Cinquanta del Novecento nelle province già italiane dell'Adriatico orientale.

\footnotetext{
${ }^{1}$ Per una panoramica generale sul problema delle violenze di massa, solitamente conosciute come "foibe giuliane" ("foibe" è il nome delle voragini carsiche diffuse in tutta l'area di frontiera tra Italia, Slovenia e Croazia in cui vennero precipitati i corpi di molte delle vittime), come pure sul dibattito storiografico e sull'uso politico della questione, si vedano Giampaolo Valdevit, Foibe. Il peso del passato, Venezia Giulia 1943-1945, Venezia, Marsilio, 1997, con saggi di Raoul Pupo, Roberto Spazzali, Nevenka Troha, Giampaolo Valdevit; Darko Dukovski, Rat i mir istarski: model povijesne prijelomnice (1943-1955), Pola, C.A.S.H., 2001; Raoul Pupo, Roberto Spazzali, Foibe, Milano, Bruno Mondadori, 2003; Elio Apih, Le foibe giuliane, Gorizia, Leg, 2010. In particolare sulle violenze della primavera del 1945: Raoul Pupo, Trieste '45, Roma-Bari, Laterza, 2010. Per un diverso punto di vista vedi Jože Pirjevec, Foibe. Una storia d'Italia, Torino, Einaudi, 2009; Luisa Accati, Renate Cogoy (a cura di), Il perturbante nella storia. Le foibe: uno studio di psicopatologia della ricezione storica, Verona, QuiEdit, 2010.
} 
Per la verità, dopo le incandescenti polemiche del decennio precedente, a partire dagli anni Sessanta del secolo scorso la questione scomparve praticamente dall'attenzione degli storici italiani e jugoslavi, per ragioni più politiche che scientifiche. I due paesi confinanti infatti, superati gli antagonismi del passato, avevano imboccato decisamente la via della distensione ${ }^{2}$ : e così, mentre i governi sperimentavano forme sempre più profonde di collaborazione, gli studiosi di storia preferivano evitare la discussione su di un tema che da parte jugoslava veniva considerato una provocazione, per esplorare invece altri momenti delle relazioni fra i due paesi e i due popoli, alla luce di un sentire antifascista comune, anche se variamente declinato. Si è trattato dunque di un classico caso di non soluzione, bensì di rimozione di un problema all'epoca intrattabile. Ciò significa che sottoterra la questione continuava a ribollire, pronta a erompere come un geyser quando la struttura esterna di contenimento fosse venuta meno. Questo è quanto accadde naturalmente dopo il 1989, così come per altre vicende oscurate dai vincoli dell'Europa divisa ${ }^{3}$. Le modalità di gestione del problema e il rapporto in tal modo determinatosi fra politica e storia appaiono decisamente interessanti.

In una prima fase, prevalse decisamente la volontà comune, in Italia e Jugoslavia e poi in Slovenia e Croazia, di evitare che il riaccendersi dell'attenzione su alcune zone oscure dei rapporti bilaterali potesse scatenare in Italia un'ondata di revisionismo storiografico politicamente assai connotato in senso nazionalista, se non addirittura neofascista. Di ciò si avevano le prima avvisaglie con la proposta, avanzata da alcuni esponenti di estrema destra, di costituire una Commissione parlamentare d'inchiesta sulle foibe ${ }^{4}$. L'unilateralità di tale approccio e le conseguenti, inevitabili strumentalizzazioni polemiche avrebbero certamente danneggiato le relazioni fra l'Italia e una Jugoslavia avviata sulla via del postcomunismo, ovvero con le eventuali repubbliche indipendenti di Slovenia e Croazia, qualora l'evoluzione della crisi jugoslava avesse determinato la scomparsa della repubblica federativa.

${ }^{2}$ Massimo Bucarelli, La "questione jugoslava" nella politica estera dell'Italia repubblicana (1947-1999), Roma, Aracne, 2008; Id., La politica estera italiana e la soluzione della questione di Trieste: gli accordi di Osimo del 1975, "Qualestoria", 2013, n. 2, pp. 29-54; Raoul Pupo (a cura di), Osimo, il punto sugli studi, numero monografico di "Qualestoria", 2013, n. 2; Massimo Bucarelli, Luca Micheletta, Luciano Monzali, Luca Riccardi (a cura di), Italy and Tito's Yugoslavia in the Age of International Détente, Bruxelles, Peter Lang, 2016.

${ }^{3}$ All'interno del ricchissimo dibattito sul tema, vedi, anche per i riferimenti bibliografici, il saggio di Guri Schwarz Tra resistenze e collaborazionismi. Considerazioni sul dibattito internazionale a settant'anni dalla Seconda guerra mondiale, in Diego D'Amelio, Patrick Karlsen (a cura di), Collaborazionismi, guerre civili e resistenze, numero monografico di "Qualestoria", 2015, n. 2, pp. 193-203.

${ }^{4}$ Nonostante la costituzione delle commissioni miste italo-slovena e italo-croata, la proposta di dar vita anche a una Commissione d'inchiesta parlamentare sulle foibe sarebbe stata effettivamente presentata nel corso di diverse legislature, ma non venne mai presa in considerazione, vista l'esistenza delle commissioni bilaterali. 
Pertanto, il 24 settembre 1990 il Consiglio comunale di Trieste — città simbolo della contesa italo-jugoslava - guidato da una coalizione di centro-sinistra, votò sorprendentemente all'unanimità (e quindi anche con il consenso dell'estrema destra) una mozione in cui si chiedeva la costituzione di una Commissione bilaterale italo-jugoslava formata da storici dei due paesi e incaricata di far chiarezza sul problema delle foibe ${ }^{5}$. L'idea venne fatta propria dal governo italiano (con il quale era stata concordata), che avviò di conseguenza i negoziati con quello di Belgrado. La trattativa fu alquanto accidentata, dal momento che nel bel mezzo la Jugoslavia si dissolse, ma venne proseguita parallelamente con i nuovi governi di Lubiana e di Zagabria e si concluse positivamente. Così, nell'ottobre 1993, con uno scambio di note fra i tre governi, vennero costituite dai rispettivi ministeri degli Esteri due commissioni miste storico culturali, una italo-slovena e una italo-croata. Su richiesta jugoslava prima e slovena e croata poi, l'originaria proposta di concentrare l'attenzione sulla questione delle foibe venne significativamente modificata: il mandato infatti fu quello di "effettuare una globale ricerca e disamina di tutti gli aspetti rilevanti delle questioni politiche e culturali bilaterali nel corso di questo secolo", secondo un approccio sicuramente assai più sensato dal punto di vista storiografico, oltre che più equilibrato da quello politico.

Le due commissioni dunque nacquero gemelle, ma ebbero destini assai diversi. Quella italo-slovena infatti ha concluso il suo operato nell'anno 2000 con un Rapporto finale congiunto, mentre la seconda ha sospeso quasi subito i suoi lavori, mai più ripresi. Formalmente, non è mai stata sciolta e nel corso degli anni vi è stato qualche timido tentativo di rianimarla, subito però abbandonato. Tale diversità di esiti va posta in relazione non solo con l'iter dei rapporti fra Italia e Croazia, ma con gli sviluppi del dibattito politico all'interno della Croazia medesima e della sua storiografia. Per quel che si può intuire, a motivare la delegazione croata a sospendere di fatto i lavori potrebbero aver contribuito in misura ragguardevole le incertezze nella valutazione dell'esperienza storica dello Stato indipendente di Croazia a guida ustaša e dei suoi rapporti con l'Italia fascista.

A ogni modo, la bipartizione della Commissione ha avuto effetti negativi sul piano interpretativo: notevoli problemi infatti la Commissione italo-slovena ha incontrato tutte le volte in cui l'analisi dei rapporti italo-sloveni andava inquadrata nell'ambito delle relazioni fra soggetti statuali. Comprensibilmente, la delegazione slovena intendeva restringere il campo ai soli rapporti fra italiani e sloveni maturati nei territori del loro insediamento storico, ma è evidente che nella gestione della cosiddetta "questione adriatica", di cui i territori abitati da sloveni costituivano solo un segmento, per il governo di Roma gli interlocutori erano stati prima Vienna e poi Belgrado, ma non certo Lubiana. Saldare la di-

\footnotetext{
${ }^{5}$ Per il testo si veda "Quaderni del Centro studi Ezio Vanoni”, 1990, n. 20-21.
} 
mensione regionale, quella nazionale e quella statuale non si è quindi rivelato affatto semplice.

Per esempio, da parte italiana la Venezia Giulia è stata sempre considerata come una realtà unitaria e di conseguenza la storiografia italiana ha affrontato globalmente i problemi posti vuoi dalla politica fascista nei confronti delle popolazioni slave ivi residenti - senza distinguere tra sloveni e croati - vuoi più tardi dalla politica jugoslava nei confronti della componente italiana ${ }^{6}$. Viceversa, gli storici sloveni non ritenevano di doversi impegnare in un confronto sulle scelte politiche compiute dalle autorità jugoslave se non per le loro ricadute sugli sloveni, e nemmeno sulla situazione creatasi nel secondo dopoguerra nella maggior parte dell'Istria annessa alla Croazia: di conseguenza, una valutazione generale di un fenomeno come quello dell'esodo degli italiani (che coinvolse anche, ma solo in misura ridotta, i territori passati sotto il controllo sloveno) diventava del tutto impossibile ${ }^{7}$. Ancora, alquanto problematico si è ri-

${ }^{6}$ Il termine "Venezia Giulia" fu coniato nel 1863 dal glottologo goriziano Graziadio Isaia Ascoli per definire in maniera unitaria tutti i territori appartenenti all'Impero asburgico rivendicati dall'Italia al suo confine orientale, divisi a quel tempo in varie circoscrizioni amministrative ma unificati, agli occhi degli italiani, dalla presenza di popolazioni parlanti dialetti di origine veneta. In tale prospettiva, la presenza sul medesimo territorio anche di popolazioni slave appariva del tutto irrilevante, e ancora più la distinzione fra sloveni e croati, generalmente ignorata anche dagli italiani residenti nella regione. Da parte jugoslava dopo il 1918 e soprattutto durante le conferenze della pace venne utilizzato il termine Julijska Krajina o, più frequentemente, la versione francese di Marche Julienne. Naturalmente, la storiografia jugoslava e, a maggior ragione, quelle slovena e croata, distinguono la realtà del "Litorale sloveno" - con cui talvolta s'intende la striscia costiera attorno a Trieste e talaltra tutta la fascia di confine con la pianura friulana - da quella dell'Istria considerata croata.

${ }^{7}$ Sul problema dell'espulsione degli italiani dalla Dalmazia e dall'Istria vedi, all'interno di una bibliografia oramai amplissima, il pionieristico Cristiana Colummi, Liliana Ferrari, Gianna Nassisi, Germano Trani, Storia di un esodo. Istria 1945-1956, Trieste, Istituto regionale per la storia del movimento di liberazione nel Friuli-Venezia Giulia, 1980, seguito da Raoul Pupo, Il lungo esodo, Milano, Rizzoli, 2005, nonché il volume collettaneo Katja Hrobat Virloget, Catherine Gousseff, Gustavo Corni (a cura di), At Home but Foreigners: Population Transfers in 20th Century Istria, Koper, Univerzitetna založba Annales, 2015. In una prospettiva comparativa vedi Marina Cattaruzza, Marco Dogo, Raoul Pupo (a cura di), Esodi. Trasferimenti forzati di popolazione nel Novecento europeo, Napoli, Esi, 2000; Guido Crainz, Raoul Pupo, Silvia Salvatici (a cura di), Naufraghi della pace. Il 1945, i profughi e le memorie divise d'Europa, Roma, Donzelli, 2008; Antonio Ferrara, Nicolò Pianciola (a cura di), L'età delle migrazioni forzate. Esodi e deportazioni in Europa 1853-1953, Bologna, il Mulino, 2012. Con un taglio di storia sociale vedi Gloria Nemec, Un paese perfetto. Storia e memoria di una comunità in esilio. Grisignana d'Istria 1930-1960, Gorizia, Leg, 1998; Ead., The Re-Definition of Gender Roles and Family Structures among Istrian Peasant Families Faced with Urban Society in Trieste (1954-1964), "Journal of Modern Italy", special issue, Gender and the Private Sphere in Italy Since 1945, 2004, n. 1, pp. 35-46; Ead., Nascita di una minoranza. Istria 1947-1965. Storia e memoria degli italiani rimasti nell'area istro-quarnerina, Rovigno, Unione italiana Fiume-Università popolare Trieste-Università degli studi di Trieste-Centro di ricerche storiche, 2012. Per una rivisitazione in chiave antropologica delle contrastanti memorie di italiani, sloveni e croati vedi Pamela Ballinger, History in Exile: Memory and Identity at the Borders of the Balkans, Princeton-Oxford, Princeton U.P., 2003. Sulla situazione specifica del Capodistriano, attualmente parte del- 
velato esprimere un giudizio storico condiviso sulle conseguenze del trattato di pace del 1946: per l'Italia si trattò di un evento catastrofico, perché la maggior parte della Venezia Giulia venne trasferita alla Jugoslavia, mentre all'interno della storiografia slovena serpeggiava un giudizio secondo il quale l'annessione dell'Istria alla Croazia sarebbe stata in qualche modo "pagata" dagli sloveni con la rinuncia a Trieste. Le specifiche difficoltà incontrate su questo punto dalle delegazioni italiana e slovena si inseriscono naturalmente nel contesto più ampio dei problemi metodologici sollevati dalla dissoluzione della Jugoslavia e dalla tendenza delle storiografie nazionalizzanti degli stati successori a rileggere l'esperienza jugoslava non solo in termini fortemente critici, ma addebitando spesso, vuoi alla dimensione federale, vuoi all'azione delle altre etnie, le responsabilità maggiori delle pagine più oscure della storia jugoslava del dopoguerra.

Infine, ma si tratta forse dell'aspetto più grave, la frammentazione dell'area jugoslava e della relativa storiografia, ha reso particolarmente arduo seguire la prospettiva analitica che la miglior storiografia italiana aveva indicato già da molti anni: vale a dire, la necessità di tenere lo sguardo largo, al di là delle beghe fra alcune centinaia di migliaia di italiani e slavi di confine, per guardare alla proiezione centro-europea della politica estera fascista, delle logiche di oc-

la Repubblica di Slovenia, vedi i molti contributi di Nevenka Troha, fra i quali Oris položaja v Koprskem okraju cone B Julijske krajine v letih 1945-1947, "Prispevki za novejšo zgodovino", 1996, n. 1-2; Politični položaj v koprskem okraju cone B Slovenskega tržaškega ozemljav prvem letu po objavi resolucije Informbiroja (1948-1949), "Prispevki za novejšo zgodovino", 1999, n. 2; Preselitve v Julijski krajini po drugi svetovni vojni, "Prispevki za novejšo zgodovino", 2000, n. 1; Ukrepi jugoslovanskih oblasti v conah B Julijske krajine, in Svobodnega tržaškega ozemlja in Italijani na Koprskem, "Annales. Series historia et sociologia", 2000, n. 1, pp. 203-216; Volitve v Okraju Koper cone B Svobodnega tržaškega ozemlja, "Prispevki za novejšo zgodovino", 2002, n. 3; Epuracija v coni B Slovenskega primorja in koprskem okraju cone B Svobodnega tržaškega ozemlja (1945-1950), "Prispevki za novejšo zgodovino", 2003, n. 2; vedi inoltre il convegno "Le ragioni e le conseguenze dell'esodo dall'Istria slovena dopo la seconda guerra mondiale / Vzroki in posledice izseljevanja iz slovenske Istre po drugi svetovni vojni", tenutosi a Capodistria nel giugno 2000 nell'ambito del Progetto interreg II/Phare CBC Italia-Slovenia; il volume Dopoguerra di confine / Povojni čas ob meji, Progetto interreg IIIA/Phare CBC Italia- Slovenia, Trieste, 2007; Jure Gombač, Esuli oppure optanti? Il caso storico alla luce della teoria moderna, Trieste, Università degli studi di Trieste, 2007. Per i pochi contributi da parte della recente storiografia croata vedi soprattutto le opere di Darko Dukovski, Rat i mir istarski, cit.; Dva egzodusa: hrvatski (1919-1941) i talijanski (1943-1955), in Adris Zbornik Zavoda za znanstveni i umjetnički rad HAZU u Splitu, Spalato, 2008, pp. 129-165; Međunacionalni odnosi u Istri nakon II. svjetskog rata, in Pazinski memorijal, Pisino, 2009, pp. 431-450; Represija, nezakonitosti i zločini vojnih $i$ civilnih organa vlasti u hrvatskom dijelu Istre 19451946, in Zorislav Lukić (a cura di), Represija i zločini komunističkog režima u Hrvatskoj, Zagabria, Matica hrvatska, 2012, pp. 207-267. Sui problemi dell'accoglienza dei profughi in Italia vedi, fra i molti e con diversissimo taglio, Enrico Miletto, Con il mare negli occhi. Storia, luoghi e memorie dell'esodo istriano a Torino, Milano, FrancoAngeli, 2005; Id., Istria allo specchio. Storia e voci di una terra di confine, Milano, FrancoAngeli, 2007; Sandi Volk, Esuli a Trieste. Bonifica nazionale e rafforzamento dell'italianità sul confine orientale, Udine, Kappa VU, 2010. 
cupazione italiane nei Balcani come pure, successivamente, dei disegni strategici di Tito e dei suoi collaboratori nell'ambito della Guerra fredda e delle relazioni interne al blocco comunista. Tali aperture sono rimaste perciò sacrificate nel lavoro della Commissione, ma per fortuna sono state recuperate dalla stagione di studi più recente ${ }^{8}$.

Pur con tali limiti strutturali peraltro, l'obiettivo iniziale è stato raggiunto: nella cruciale fase di transizione degli anni Novanta, il ripensamento degli aspetti più oscuri e dei rapporti fra italiani e slavi del Sud è stato affidato agli storici piuttosto che al dibattito politico e ciò ha fatto sì che la rilettura del passato non avvenisse in maniera tanto semplicistica quanto dirompente, bensì in forma critica. Certo, nel frattempo tentativi di strumentalizzazione e polemiche non sono mancati, da varie parti, ma la possibilità di delegare al lavoro della Commissione il dibattito sui temi più controversi, ha contribuito in misura cospicua a impedire il deragliamento delle discussioni.

\section{L'operato della Commissione italo-slovena}

Entrando dunque nel merito dei lavori e dei loro risultati, il mandato originario della Commissione era quello di concentrarsi "sugli elementi positivi che uniscono i due popoli, contribuendo in tal modo a chiarire quegli eventi che hanno causato difficoltà nel passato alle loro relazioni”, dedicando opportuno spazio anche alle relazioni culturali. A tali indicazioni la Commissione si è attenuta solo in parte, per varie ragioni.

\footnotetext{
${ }^{8}$ Per quanto riguarda le politiche di occupazione italiane vedi Davide Rodogno, Il nuovo ordine mediterraneo. Le politiche di occupazione dell'Italia fascista in Europa (1940-1943), Torino, Bollati Boringhieri, 2003; Brunello Mantelli (a cura di), L'Italia fascista come potenza occupante: lo scacchiere balcanico, numero monografico di "Qualestoria", 2002, n. 1; Erik Gobetti, L'occupazione allegra. Gli italiani in Jugoslavia (1941-1943), Roma, Carocci, 2007; Id., Alleati del nemico. L'occupazione italiana in Jugoslavia (1941-1943), Roma-Bari, Laterza, 2013; Francesco Caccamo, Luciano Monzali (a cura di), L'occupazione italiana della Jugoslavia (19411943), Roma, Le Lettere, 2008. Sui rapporti fra partiti comunisti vedi almeno Marco Galeazzi, Togliatti e Tito. Tra identità nazionale e internazionalismo, Roma, Carocci, 2005; Patrick Karksen, Frontiera rossa. Il Pci, il confine orientale e il contesto internazionale 1941-1955, Gorizia, Leg, 2010; Amedeo Osti Guerrazzi, L'esercito italiano in Slovenia, 1941-1943. Strategie di repressione antipartigiana, Roma, Viella, 2011; i vari contributi di Bojan Godeša, fra i quali "Naš odnos do Italijanov naj bo miren in dostojen, toda nič več": nekateri vidiki razpetosti med nacionalnim in internacionalnim med slovenskimi komunisti na Primorskem, "Prispevki za novejšo zgodovino", 2000, n. 1; Slovenski komunisti in umestitev Trsta v program Zedinjene Slovenije med drugo svetovno vojno, "Prispevki za novejšo zgodovino", 2005, n. 2; I comunisti sloveni e la questione di Trieste durante la Seconda guerra mondiale, "Qualestoria", 2007, n. 1, pp. 119-132; Italijanska in nemška okupacija Slovenije med drugo svetovno voino — nekateri primerjalni vidiki niune začetne okupacijske politike, "Acta Histriae", 2012, n. 3, pp. 433-444, nonché Zdenko Cepic, Damijan Guštin, Nevenka Troha, La Slovenia durante la seconda guerra mondiale, Udine, Istituto friulano per la storia del movimento di liberazione, 2013.
} 
In primo luogo, nonostante la presenza tra i commissari di personalità estranee al mondo della ricerca storica - giornalisti, scrittori, da parte italiana anche il rappresentante di una delle principali organizzazioni degli esuli, l'Associazione nazionale Venezia Giulia e Dalmazia —, a prevalere sono state nettamente le competenze degli studiosi di storia politica. Se a ciò si aggiunge l'indiscussa centralità della dimensione nazionale e ideologica nei rapporti fra italiani e sloveni nel Novecento, si capisce come gli aspetti di natura culturale e sociale siano rimasti relegati al margine dei lavori.

Inoltre, la direttiva "buonista" espressa dalle diplomazie è apparsa in troppo stridente contrasto con la realtà di un periodo storico caratterizzato proprio dalla moltiplicazione e dall'esasperazione dei conflitti fra italiani e sloveni. Di fatto quindi, il lavoro della Commissione, pur senza trascurare momenti e aspetti positivi, si è concentrato prevalentemente sui motivi di conflitto sorti fra la fine dell'Ottocento e la metà del Novecento. È stato fin da subito ben chiaro come dietro tale scelta stesse la volontà dei commissari di fare i conti, seriamente e senza reticenze, proprio con i nodi sui quali in passato si erano registrati non solo orientamenti diversi - il che è normale e positivo - ma veri propri blocchi di comunicazione. Gli effetti di tale decisione sono stati però dilatati dall'orientamento, assunto in una fase iniziale dei lavori di fronte alla mole delle questioni da affrontare, di concludere l'analisi alla fine degli anni Cinquanta. In questo modo, a restar fuori dal discorso sono stati proprio quegli ultimi decenni del Novecento che erano stati caratterizzati dal netto miglioramento dei rapporti bilaterali ${ }^{9}$. A partire dagli anni Sessanta infatti, alla luce dei comuni interessi, sul piano strategico la Jugoslavia divenne per l'Italia un utilissimo cuscinetto nei confronti del Patto di Varsavia e l'Italia un fattore di sicurezza per la Jugoslavia, come emerse con evidenza durante la crisi cecoslovacca; sul piano economico l'interscambio crebbe sensibilmente; sul piano concettuale si passò dalla nozione di confine chiuso, barriera fra mondi alternativi, alla formula del "confine-ponte", capace di costituire una risorsa di posizione di cui si sarebbe potuta giovare la struttura economica e insediativa delle regioni contermini ${ }^{10}$; sul piano locale, ciò si tradusse nell'avvio di una forte col-

${ }^{9}$ Sul piano diplomatico vedi Massimo Bucarelli, Luca Micheletta, Luciano Monzali, Luca Riccardi (a cura di), Italy and Tito's Yugoslavia in the Age of International Détente, Bruxelles, Peter Lang, 2016; per una riflessione complessiva vedi Diego D'Amelio, Frontiere in transizione. Il lungo dopoguerra dei confini italiani fra eredità, emergenze e distensioni, in Diego D'Amelio, Andrea Di Michele, Giorgio Mezzalira (a cura di), La difesa dell'italianità. L'Ufficio per le zone di confine a Bolzano, Trento e Trieste (1945-1954), Bologna, il Mulino, 2015, pp. 539-594.

${ }^{10}$ Giorgio Valussi, Il confine nordorientale d'Italia, Trieste, Lint, 1972; Milan Bufon, Sviluppo etnico e regionale delle aree di confine: il caso degli Sloveni nel Friuli-Venezia Giulia, Trieste, Quaderni SLORI, 1990; Id., Cultural and Social Dimension of Borderlands: the Case of the Italo-Slovene Trans-Border Area, "Geojournal”, 1993, n. 3, pp. 235-240; Moreno Zago, Il confine-ponte: la strategia, in Georg Meyr, Raoul Pupo, (a cura di), Dalla cortina di ferro al confine ponte. A cinquant'anni dal Memorandum di Londra, l'allargamento della Nato e dell'Unione Europea, Trieste, Edizioni Comune di Trieste, 2008. 
laborazione transfrontaliera, come quella realizzata a partire dal 1978 dalla Comunità di lavoro Alpe Adria, vero modello di dialogo fra ragioni allora appartenenti a sistemi economici e regimi politici diversi ${ }^{11}$.

Rispettato invece dai commissari è stato il mandato di compiere una ricognizione critica comune sulla storiografia esistente piuttosto che di indicare nuove piste di ricerca e, in linea con tale orientamento, anche i contenuti del rapporto appaiono più prudenti che innovativi. Pure in questo caso, le motivazioni della scelta sono diverse.

Prima di tutto - ragione tanto banale quanto efficace - il budget a disposizione delle Commissione copriva con una certa fatica le spese delle sessioni bilaterali, ma non contemplava alcuna voce attinente alla ricerca. Di ricerche in quegli anni i commissari ne hanno fatte ugualmente, anche in collaborazione fra di loro, ma sui loro finanziamenti e su temi specifici, non all'interno di un piano complessivo. In secondo luogo, i componenti della Commissione erano preoccupati, più che d'inventare nuovi paradigmi, di offrire giudizi equilibrati sui molti temi che sino a quel momento non solo erano stati oggetto di forti dispute fra gli storici, ma avevano anche costituito l'occasione per un massiccio e spregiudicato uso politico della storia. Inoltre, pesava non poco sulle discussioni, la diversità dei punti di partenza, riassumibili nella constatazione che, mentre la storiografia italiana aveva fatto già da tempo i conti con il fascismo, quella slovena doveva appena cominciare a farli con il comunismo.

Esistevano così vasti terreni di dibattito già ampiamente dissodati, come quelli relativi al fascismo di confine, ai tentativi di "bonifica etnica" della minoranza slovena compiuti negli anni Venti e Trenta da parte delle autorità italiane, alle politiche di occupazione italiana in Slovenia, alla collaborazione fra italiani e sloveni durante la lotta di liberazione. A questi si accostavano altrettante zone oscure, relative non soltanto a singoli episodi particolarmente tragici e controversi - come quello delle foibe - ma, in termini assai più generali - agli aspetti articolati e contraddittori della lotta di liberazione, al ruolo del collaborazionismo sloveno nella provincia di Lubiana e nel Litorale, alla complessità dei rapporti fra i movimenti resistenziali e gli stessi partiti comunisti, all'intreccio fra spinte nazionali, lotta di classe e politica di potenza all'interno del movimento partigiano e del nuovo Stato comunista jugoslavo, al legame fra le pratiche estreme di violenza messe in atto durante e dopo la guerra e le finalità e strutture del nuovo regime in via di instaurazione nei territori a qualsiasi titolo ritenuti jugoslavi.

Il percorso da compiere da parte delle due storiografie rappresentate nella Commissione era dunque fortemente asimmetrico e, di fronte a questa situa-

\footnotetext{
${ }^{11}$ Alpe Adria. Una regione europea, Milano, Ispi, 1988; Liviana Poropat, Alpe Adria e iniziativa centro-europea, Napoli, Esi, 1993, Andreas Moritsch, Alpen-Adria. Zur Geschichte einer Region, Klagenfurt, Mohorjeva, 2001; Marco Antonsich, Il Nord-Est tra Mitteleuropa e Balcani: il caso del Friuli-Venezia Giulia, in Id. e al., Geopolitica della Crisi. Balcani, Caucaso e Asia centrale nel nuovo scenario internazionale, Milano, Egea, 2002, pp. 141-248.
} 
zione, l'intento principale dei commissari è stato di taglio minimalista, consistente nella ricerca di un linguaggio comune e di un terreno solido di consenso su di alcune linee guida interpretative. Ciò si è rivelato più difficile in riferimento ai ragionamenti di lungo periodo, ai giudizi complessivi sui rapporti fra i due popoli, che non a singoli momenti della storia del Novecento, per quanto apparentemente più controversi. Il fatto è che, attorno ad alcune questioni particolarmente spinose - come quella delle foibe - alcuni commissari italiani e sloveni stavano già lavorando in contatto fra loro da alcuni anni sulle medesime fonti. Non è risultato quindi troppo arduo tradurre gli esiti di tali ricerche in formulazioni nuove rispetto agli schematismi del passato e capaci di dar conto dei diversi piani di lettura di quella tragica stagione.

Molto più problematico, invece, è stato raggiungere un consenso tale da materializzarsi in un testo condiviso sui cosiddetti "capisaldi interpretativi" cui la delegazione slovena è sembrata per lungo tempo volersi ancorare, in piena continuità con la precedente tradizione jugoslava. Materia principale del contendere era una visione lineare del processo storico, stretta da cogenti nessi causa-effetto miranti all'individuazione della "colpa primigenia" delle disgrazie novecentesche. Con il tempo e la discussione, sempre serena anche se intensa, il determinismo iniziale è stato abbandonato ed è stato possibile approdare a una visione più articolata della dinamica storica, attenta alle continuità come alle novità e capace d'individuare l'intreccio dei fili che si sono intersecati nel comporre i diversi fenomeni storici.

Un'altra incognita era legata alle stesse origini e struttura della Commissione, avente come destinatari del suo lavoro i due ministeri degli Esteri: ciò infatti comportava il rischio che l'eventuale risultato finale si configurasse come una sorta di "storia ufficiale" concordata ed edulcorata. Tale non è stato l'intento dei commissari, che si sono proposti invece di limitarsi a un lavoro preliminare ma indispensabile, quello di sgombrare il campo dalle incrostazioni ideologiche che si erano accumulate in particolare su alcuni nodi assai controversi, in modo da sottrarre la materia del contendere ai tentativi più spregiudicati di uso pubblico del passato, creando nel tempo un tessuto stabile di relazioni fra le due storiografie nazionali, fondato su di un approccio critico condiviso alla storia comune.

Così facendo, la Commissione è effettivamente pervenuta alla stesura di un Rapporto finale sottoscritto da tutti i partecipanti, anche se attraverso un percorso non privo di colpi di scena. Se infatti le sostituzioni di alcuni membri della delegazione italiana sono state dovute a motivi di salute, o sopraggiunte incompatibilità, il cambio di un componente la delegazione slovena è stato originato invece dal radicale dissenso nei confronti della linea assunta dalla delegazione medesima, giudicata troppo lontana dai precedenti paradigmi interpretativi ${ }^{12}$.

${ }^{12}$ I componenti originali della delegazione italiana della Commissione erano i professori [MAI abbreviazioni sui titoli] Sergio Bartole (che fungeva da copresidente), Elio Apih, Angelo Ara, Paola Pagnini, Fulvio Salimbeni, lo scrittore Fulvio Tomizza e il senatore Lucio Toth. 


\section{Un bilancio}

In linea generale, si può dire che l'esperienza della Commissione ha costituito la fine di una stagione piuttosto che l'inizio di una nuova. A confrontarsi sono state pur sempre due storiografie che si autoproclamavano nazionali e facevano riferimento alle istituzioni dei rispettivi stati nazione di riferimento. Inoltre, nelle due delegazioni non erano presenti storici appartenenti né alla minoranza slovena in Italia né a quella italiana in Slovenia.

Scarso spazio quindi ha avuto l'attenzione per le incertezze e contaminazioni identitarie, come pure per i processi di formazione e diffusione delle ideologie nazionali. Non a caso, il primo periodo preso in considerazione, quello antecedente alla Prima guerra mondiale, è stato risolto assai velocemente sulla scorta dei tradizionali studi italiani e sloveni, senza particolari spunti di problematizzazione. Anche nell'analisi dei periodi successivi, italiani e sloveni sono stati concepiti come gruppi nazionali ben definiti, impegnati in un rispettivo e serrato confronto.

Le prospettive di ricerca sono ora diverse, nei contenuti e nei metodi. Le identità nazionali appaiono prevalentemente quali costrutti da esplorare nel loro farsi, disfarsi e combinarsi. La dimensione comparativa, fra le vicende alto-adriatiche e quelle di altre aree europee lungo la fascia dal Baltico al Mediterraneo, costituisce oggi una priorità nelle indagini e dà sostanza alla concettualizzazione del "laboratorio giuliano" quale caso di studio particolarmente fecondo per l'analisi della contemporaneità nella transizione fra Europa centrale e mediterranea. Le indagini in corso si inseriscono nel più ampio e promettente filone di studi dei borderlands, categoria che ha permesso di legare assieme le esperienze postimperiali di territori fortemente plurali ${ }^{13}$. Il futuro della collaborazione italo-slovena ormai non va cercato più nel confronto fra storici della nazione, quanto nell'affermarsi di una storiografia postnazionale, attraverso la quale studiosi italiani e sloveni mostrino la loro capacità di entrare nelle sensibilità altrui, di smontare i meccanismi delle appartenen-

In un secondo momento Bartole venne sostituto (anche nella copresidenza) dal professor Giorgio Conetti; Fulvio Tomizza venne sostituito dal professor Raoul Pupo e Elio Apih dalla professoressa Marina Cattaruzza. I componenti originali della delegazione slovena erano la dottoressa Milica Kacin-Wohinz (che fungeva da copresidente), i dottori France Dolinar, Boris Gombač, Branko Marusic, Boris Mlakar, Nevenka Troha e Andrei Vovco. Dopo le dimissioni di Mlakar è subentrato il dottor Aleksander Vuga, e più tardi, in seguito alle dimissioni di Gombač, è rientrato Mlakar. Dopo l'uscita dalla Commissione, e in evidente polemica con essa, Boris Gombač ha pubblicato il volumetto Slovenija, Italija. Od preziranja do priznanja, Ljubljana, Debora, 1996. Sui lavori della Commissione e il loro significato vedi anche Milica Kacin Wohinz, La Commissione italo-slovena alla ricerca di un passato comune, in Simonetta Soldani (a cura di), Enzo Collotti e l'Europa del Novecento, Firenze, Firenze University Press, 2011.

${ }^{13}$ Vedi come riferimento Alexander V. Prusin, The Lands Between: Conflicts in the East European Borderlands, Oxford, Oxford U.P., 2010. 
ze scontate, di verificare come questi si siano creati e quali esperienze abbiano trascurato ${ }^{14}$.

Il lascito più importante della Commissione va quindi individuato su due piani. Il primo, la puntualizzazione, in chiave critica e rigorosamente fondata, di alcune questioni cruciali — quali appunto quelle relative alle stagioni della violenza — che stavano all'origine della Commissione stessa, rispetto alla quali le valutazioni espresse nel Rapporto finale sono divenute un punto di riferimento ineludibile, se pur sempre migliorabile. Per quanto riguarda ad esempio la questione delle foibe, che abbiamo visto aver dato origine alla Commissione, il Rapporto si esprime in maniera articolata ma assai chiara, non sottraendosi al confronto con le categorie interpretative più frequentemente utilizzate, magari in contrapposizione fra loro, nei dibattiti scientifici e pubblici sull'argomento, come le coppie "liberazione/occupazione" e "resa dei conti/progetto preordinato", o i concetti di "epurazione preventiva" e "violenza di Stato". Ecco il testo:

L'estensione del controllo jugoslavo dalle aree già precedentemente liberate dal movimento partigiano fino a tutto il territorio della Venezia Giulia fu salutata con grande entusiasmo dalla maggioranza degli sloveni e dagli italiani favorevoli alla Jugoslavia. Per gli sloveni si trattò di una duplice liberazione, dagli occupatori tedeschi e dallo Stato italiano. Al contrario, i giuliani favorevoli all'Italia considerarono l'occupazione jugoslava come il momento più buio della loro storia, anche perché essa si accompagnò nella zona di Trieste, nel Goriziano e nel Capodistriano ad un'ondata di violenza che trovò espressione nell'arresto di molte migliaia di persone, - in larga maggioranza italiane, ma anche slovene contrarie al progetto politico comunista jugoslavo - , parte delle quali vennero a più riprese rilasciate; in centinaia di esecuzioni sommarie immediate - le cui vittime vennero in genere gettate nelle "foibe"; nella deportazione di un gran numero di militari e civili, parte dei quali perì di stenti o venne liquidata nel corso dei trasferimenti, nelle carceri e nei campi di prigionia (fra i quali va ricordato quello di Borovnica), creati in diverse zone della Jugoslavia.

Tali avvenimenti si verificarono in un clima di resa dei conti per la violenza fascista e di guerra ed appaiono in larga misura il frutto di un progetto politico preordinato, in cui confluivano diverse spinte: l'impegno ad eliminare soggetti e strutture ricollegabili (anche al di là delle responsabilità personali) al fascismo, alla dominazione nazista, al collaborazionismo ed allo Stato italiano, assieme ad un disegno di epurazione preventiva di oppositori reali, potenziali o presunti tali, in funzione dell'avvento del regime comunista, e dell'annessione della Venezia Giulia al nuovo Stato jugoslavo. L'impulso primo della repressione partì da un movimento rivoluzionario che si stava trasformando in regime, convertendo quindi in violenza di Stato l'animosità nazionale ed ideologica diffusa nei quadri partigiani.

${ }^{14}$ Marta Verginella, La storia di confine tra sguardi incrociati e malintesi, in Ead. (a cura di), La storia al confine e oltre il confine. Uno sguardo sulla storiografia slovena, numero monografico di "Qualestoria”, 2007, n. 1, pp. 5-11; Ead., Asimmetrie, malintesi e sguardi speculari: da una storia etnocentrica ad una storia plurale e congiunta della regione alto-adriatica, "Acta Histriae", 2012, n. 3. Al momento peraltro, l'esempio migliore di tale prospettiva postnazionale non riguarda la dimensione italo-slovena, ma quella più ampia adriatica, quale risulta dal volume di Egidio Ivetich, Un confine nel Mediterraneo. L'Adriatico orientale tra Italia e Slavia (1300-1900), Roma, Viella, 2014. 
Il secondo aspetto da sottolineare riguarda invece lo stimolo potente al lavoro comune fra gli storici delle repubbliche di Italia e di Slovenia, posto che in precedenza soltanto alcuni di loro avevano già sperimentato forme di collaborazione all'interno di un contesto alquanto diverso: particolarmente stretti erano stati infatti, soprattutto a partire dagli anni Settanta, i rapporti fra la rete degli Istituti per la storia del movimento di liberazione in Italia e quella degli Istituti per la storia del movimento operaio in Jugoslavia, mentre altri studiosi avevano trovato qualche difficoltà a confrontarsi con i colleghi allora jugoslavi. Il risultato è stato la moltiplicazione degli scambi di fonti, dei contributi su riviste italiane e slovene, l'organizzazione di seminari e convegni in comune, la definizione di progetti di ricerca transfrontalieri ed europe ${ }^{15}$. Alcune iniziative sono andate a buon fine e altre no, com'è usuale che avvenga. Anche le convergenze e i dissensi si sono ben distribuiti in maniera trasversale, a seconda più delle opinioni e delle simpatie che non delle etnie e delle patrie. Insomma, banalità, ed è proprio in questo che sta il loro valore, nel loro essere segno di una normalizzazione dei rapporti che mancava da troppo tempo.

Tali effetti positivi risultano maggiormente chiari dal confronto con lo stato dei rapporti fra studiosi italiani e croati, che non hanno beneficiato dell'esperienza della Commissione. Da vent'anni almeno a questa parte, quei rapporti si sono ridotti al lumicino, nonostante l'arco problematico su cui lavorare assieme sia ben più vasto. Rimane comunque la speranza che dopo l'ingresso della Croazia nell'Unione europea, quel che non poterono volontà politiche e propensioni intellettuali degli storici dei due paesi, forse potranno i finanziamenti dei progetti "Interreg".

La consegna nell'anno 2000 del Rapporto finale della Commissione italoslovena è caduta peraltro in una fase politica diversa rispetto al momento in cui l'operazione bilaterale era stata varata. In Italia le paventate speculazioni dell'estrema destra nazionalista si sono rivelate decisamente meno rilevanti rispetto a un altro processo, imprevedibile al momento della costituzione della Commissione: il crollo cioè della cosiddetta "prima repubblica", la scomparsa dalla scena politica della Democrazia cristiana - che del precedente assetto era stata il perno - , la corsa alla sua successione e quindi la ricerca di legittimazione da parte degli eredi delle compagini politiche che l'egemonia democristiana, combinata con i vincoli della Guerra fredda, aveva mantenuto fuori dall'area di governo e cioè i comunisti e i neofascisti. Tali eredi dunque, impegnati in faticosi

${ }^{15}$ Fra le riviste, si segnalano in particolare "Qualestoria" (di cui vanno ricordati anche i due numeri monografici 2007, n. 1, e 2016, n. 1, dedicati entrambi alla storiografia slovena), "Storia contemporanea in Friuli", "Prispevki za novejšo zgodovino"; "Annales. Series historia et sociologia", "Acta Histriae". Per quanto riguarda le comuni iniziative di ricerca vedi i già cit. Le ragioni e le conseguenze dell'esodo dall'Istria slovena dopo la seconda guerra mondiale / Vzroki in posledice izseljevanja iz slovenske Istre po drugi svetovni vojni; e Dopoguerra di confine I Povojni čas ob meji, nonché Katja Hrobat Virloget, Catherine Gousseff, Gustavo Corni (a cura di), At Home but Foreigners. 
processi di ricostruzione delle loro identità e animati da propositi convergenti, hanno pensato bene di utilizzare la memoria delle tragedie italiane del confine orientale come uno dei principali terreni per lo scambio della reciproca legittimazione. Esemplare sotto questo profilo è stato l'incontro fra l'allora presidente della Camera dei deputati ed esponente del Partito democratico della sinistra, Luciano Violante, e il leader di Alleanza nazionale Gianfranco Fini, avvenuto al Teatro Verdi di Trieste - tradizionalmente considerato il "tempio dell'italianità giuliana" - nel marzo 1998.

Al riguardo, sono stati espressi giudizi assai diversi: vi è chi fra gli studiosi ha accusato con parole di fuoco la sinistra italiana di essersi venduta al nazionalismo e chi invece ha visto in quell'incontro un progresso sostanziale per la democrazia italiana ${ }^{16}$. Senza entrare nel merito, non sembra dubbio che i nuovi assetti politici del paese abbiamo influito sullo scarso entusiasmo con cui in Italia il committente ha accolto il rapporto finale della Commissione, che poco concedeva alla mitologie nazionali e ideologiche. Il governo italiano non l'ha fatto proprio, limitandosi a consentirne la diffusione mediante i consueti canali scientifici: una decisione formalmente ineccepibile e per certi versi opportuna, nel senso di una presa di distanza della politica dalla storia, ma certo in contraddizione con le aspettative che legavano un salto di qualità nei rapporti tra paesi confinanti alla capacità di far chiarezza sulle ombre del loro passato.

\section{L’istituzione del Giorno del ricordo}

Che la temperie politica fosse cambiata, lo testimonia del resto ad abundantiam l'adozione da parte italiana della legge 30 marzo 2004, n. 92, che "riconosce il 10 febbraio - anniversario della firma del trattato di pace del 1947 quale "Giorno del ricordo" al fine di conservare e rinnovare la memoria della tragedia degli italiani e di tutte le vittime delle foibe, dell'esodo dalle loro terre degli istriani, fiumani e dalmati nel secondo dopoguerra e della più complessa vicenda del confine orientale" ${ }^{17}$. La decisione è arrivata dopo un iter legislativo piuttosto interessante da esaminare, cominciato ancora nel 1995 con la proposta da parte di alcuni deputati di Alleanza nazionale di un disegno di legge riguardante la "Concessione di un riconoscimento ai congiunti degli infoibati", che in realtà mirava a ottenere da parte dello Stato italiano un riconoscimen-

\footnotetext{
${ }^{16}$ Per i due opposti punti di vista vedi ad esempio, J. Pirjevec, Foibe, cit., e Liborio Mattina, Democrazia e nazione: dibattito a Trieste tra Luciano Violante e Gianfranco Fini, Trieste, Eut, 1998. Vedi inoltre al riguardo i commenti di Pamela Ballinger, in Exhumed Histories: Trieste and the Politics of (Exclusive) Victimhood, "The Journal of Southern Europe and the Balkans", 2004, n. 2, pp. 145-159, qui p. 156.
}

17 "Gazzetta ufficiale", 13 aprile 2004, n. 86. 
to alla memoria, più che dei civili uccisi nelle ondate di violenza del 1943 e del 1945, dei militari della Rsi caduti al confine orientale ${ }^{18}$. La proposta non venne nemmeno discussa nel corso della legislatura, ma venne ripresa in quella successiva e ottenne l'approvazione della Camera, dopo che il dibattito parlamentare, grazie anche alla consulenza di alcuni storici appartenenti alla rete degli Istituti per la storia del movimento di liberazione, aveva consentito di escludere dal riconoscimento i caduti in combattimento - che con le "foibe", così come definite nella letteratura scientifica, non avevano nulla a che spartire - nonché i criminali di guerra ${ }^{19}$. Nell'ultimo giorno della legislatura invece, l'8 marzo 2011, la proposta venne bocciata dal Senato.

Gli anni seguenti registrarono una svolta sia nei contenuti delle proposte legislative riguardanti le vicende del confine orientale, sia nella loro capacità di attrarre un consenso quasi plebiscitario tra le forze politiche. La nuova legislatura infatti vide il fiorire di varie proposte di legge volte a istituire una giornata della memoria delle foibe e dell'esodo ${ }^{20}$. L'unificazione dei diversi progetti e il dibattito parlamentare condussero alla redazione di un testo che, formalmente, riguardava appunto l'istituzione del Giorno del ricordo, ma che nell'articolato riproponeva anche la concessione di un'"insegna metallica con relativo diploma" ai congiunti degli infoibati nella Venezia Giulia e in Dalmazia (compresi quindi alcuni dei territori annessi dopo l'aggressione alla Jugoslavia dell'aprile 1941), con la sola esclusione dei caduti in combattimento. Inoltre, la nuova formulazione limitava fortemente - sino a renderli di fatto inapplicabili - i caveat che nelle precedenti versioni erano stati inseriti con il preciso intento di impedire l'attribuzione di un pubblico riconoscimento anche a criminali di guerra e ai membri di formazioni militari e di polizia ben note per il comportamento efferato tenuto nei confronti dei resistenti, sia italiani che jugoslavi nella Venezia Giulia, come per esempio l'Ispettorato speciale di pubblica sicurezza. Ciò nonostante, grazie alla nuova impostazione che poneva l'accento sulla creazione di una giornata memoriale, la legge venne approvata con una schiacciante maggioranza e dichiarazioni di voto favorevoli anche da par-

18 Proposta di legge d'iniziativa dei deputati Menia e altri, 11 luglio 1995: si veda all'indirizzo web http://legislature.camera.it/_dati/leg12/lavori/stampati/pdf/56004.pdf., ultimo accesso 10 ottobre 2016.

${ }_{19}$ Proposta di legge Menia: Concessione di un riconoscimento ai congiunti degli infoibati (1563) ed abbinata proposta di legge: Di Bisceglie (6724), in A.P., Camera dei deputati, XIII leg., allegato ai Resoconti, Seduta $1^{\circ}$ marzo 2001, n. 870, all'indirizzo web http://leg13.camera. it/_dati/leg13/lavori/stenografici/sed870/pdfa003.pdf, ultimo accesso 10 ottobre 2016.

${ }^{20}$ Proposta di legge: Istituzione del "giorno della memoria e della testimonianza" in ricordo delle terre d'Istria, di Fiume e della Dalmazia, nonché degli esuli giuliano-dalmati, all'indirizzo web http://legxiv.camera.it/_dati/leg14/lavori/schedela/trovaschedacamera_wai.asp?PDL=3661, ultimo accesso 10 ottobre 2016; Disegno di legge: Istituzione del "giorno della memoria" dell'esodo di istriani, fiumani e dalmati, all'indirizzo web www.senato.it/service/PDF/PDFServer/ DF/103627.pdf, ultimo accesso 10 ottobre 2016. 
te di esponenti della minoranza slovena in Italia eletti nelle file dei Democratici di sinistra (ex Pci) ${ }^{21}$.

Lo spirito di conciliazione nazionale prevalse dunque largamente in quell'occasione sulla prudenza suggerita dagli storici e in effetti nel seguente decennio nessuno è sembrato prestare attenzione ai lavori della Commissione costituita presso la Presidenza del consiglio allo scopo di vagliare le richieste di riconoscimento presentate dai congiunti degli infoibati, che nel primo decennio dall'entrate in vigore della legge sono state circa 300, cifra verosimilmente assai inferiore alle previsioni. A dieci anni di distanza invece, alcuni casi emersi con un certo clamore sembrano suggerire che i riconoscimenti siano stati elargiti con notevole liberalità, vuoi a militari di Salò caduti in combattimento contro i partigiani, vuoi a uomini delle istituzioni e soldati del regio esercito accusati da parte jugoslava di crimini di guerra. Al riguardo, particolare rilievo assumono i casi del capitano Paride Mori, ufficiale del battaglione M della Rsi, caduto in un agguato partigiano in val Baccia, presso Gorizia, nel febbraio 1944, e di Vincenzo Serrentino, ultimo prefetto italiano della provincia di Zara, arrestato dagli jugoslavi a Trieste nella primavera del 1945 e fucilato a Sebenico nel maggio 1947 come criminale di guerra, per aver fatto parte del Tribunale straordinario della Dalmazia che nell'autunno del 1941 comminò numerose condanne a morte nei confronti di presunti aderenti al movimento partigiano jugoslavo ${ }^{22}$. Il caso Mori ha creato un certo imbarazzo fra le associazioni dei profughi giuliani e dalmati - principali sostenitrici del Giorno del ricordo alcune delle quali si sono affrettate a prendere le distanze dalla prassi adottata dalla Commissione, mentre altre invece hanno difeso a spada tratta le scelte compiute dai commissari ${ }^{23}$. Diversamente, la memoria di Serrentino è tuttora oggetto di venerazione negli ambienti della diaspora giuliano-dalmata.

Che dunque qualcosa di grave sarebbe prima o poi accaduto, era facile prevedere. In ogni caso, l'eco delle polemiche è stato piuttosto limitato, rimanendo in genere circoscritto agli ambienti intellettuali e politici che costantemente se-

${ }^{21}$ Per quanto riguarda le discussioni alla Camera dei deputati, si veda in particolare A.P., Camera dei deputati, XIV leg., Discussioni, Seduta 11 febbraio 2004, n. 422, all'indirizzo web http://documenti.camera.it/_dati/leg14/lavori/stenografici/sed422/pdfs001.pdf, ultimo accesso 10 ottobre 2016; per l'intero percorso della proposta di legge al Senato si veda Atto Senato n. 2752. XIV legislatura all'indirizzo web www.senato.it/leg/14/BGT/Schede/Ddliter/20980.htm, ultimo accesso 10 ottobre 2016.

${ }^{22}$ Sul caso di Paride Mori si vedano, fra gli altri, gli articoli comparsi sul "Corriere della sera" del 17 marzo 2015, e su "La Repubblica" e "Il Fatto quotidiano" del 25 aprile 2015. Sul caso Serrentino si veda Filippo Focardi, Rielaborare il passato. Usi pubblici della storia e della memoria in Italia dopo la prima repubblica, in Giorgio Resta, Vincenzo Zeno-Zencovich (a cura di), Riparare, risarcire, ricordare. Un dialogo tra storici e giuristi, Napoli, Editoriale scientifica, 2012, p. 260.

${ }^{23}$ Vedi al riguardo le differenti prese di posizione comparse rispettivamente su "L'Arena di Pola" e "Coordinamento adriatico" nell'estate 2015. 
guono le modalità di gestione del Giorno del ricordo ${ }^{24}$; la Commissione ha tacitamente concluso il suo lavoro - anche se vi sono spinte per riattivarla - e non si sa se i materiali istruttori raccolti verranno prima o poi messi a disposizione degli studiosi.

A prescindere comunque da ciò, la giornata memoriale ha registrato un grande successo. Nel corso di un decennio si sono impegnate nella sua valorizzazione le principali istituzioni dello Stato italiano, dalla Presidenza della Repubblica agli enti locali, da piccoli comuni fino a consigli rionali di innumerevoli città, oltre a scuole di ogni ordine e grado, quasi tutte concentrate nel Centronord del paese, mentre a sud di Roma le tematiche legate alla storia dell'area nord-adriatica e dei rapporti fra l'Italia e i suoi vicini orientali hanno suscitato in genere scarso interesse.

L'ampiezza dello schieramento favorevole alla legge e lo sforzo profuso dalle forze politiche di centro-sinistra hanno fatto sì che la celebrazione non fosse monopolizzata dall'estrema destra nazionalista. Non che siano mancate le strumentalizzazioni, fra le quali esemplare risulta la campagna condotta per alcuni anni da Alleanza nazionale - al tempo partito di governo nelle coalizioni di centro-destra - per l'intitolazione di vie e piazze di ogni comune d'Italia ai "martiri delle foibe". Nei primi anni inoltre, le associazioni dei profughi hanno fatto quanto in loro potere affinché l'attenzione suscitata dalla giornata si concentrasse esclusivamente sulla vicenda delle foibe e su quella dell'esodo, mentre i mezzi di comunicazione - che improvvisamente avevano scoperto una storia pressoché inedita, ma che interessava alla politica - hanno puntato i loro riflettori principalmente sulla questione delle foibe, che meglio di ogni altra sembrava prestarsi a drammatizzazioni e semplificazioni.

Infine, la data stessa scelta per la celebrazione - il 10 febbraio, giorno in cui nel 1947 venne firmato il trattato di pace con l'Italia che prevedeva la cessione alla Jugoslavia di Zara, di Fiume e di quasi tutta l'Istria - portava insita in sé una forzatura tutt'altro che lieve, sulla quale i parlamentari avevano preferito sorvolare. Non c'è dubbio infatti che nella memoria dei profughi giuliano-dalmati la firma del trattato di pace sia sempre stata considerata come l'evento fondante della loro tragedia, mentre date alternative, che pur erano state proposte, non possedevano alcun valore simbolico riconosciuto. Ma è anche vero che quell'opzione poteva - e, nei desideri di taluno, voleva - ridare fiato a quanti, coerentemente con la tradizione nazionalista, imputavano alla "debolez-

\footnotetext{
${ }^{24}$ È questo per esempio il caso dei siti www.nuovaalabarda.org; www.diecifebbraio.info; www.wumingfoundation.com/giap; www.cnj.it/documentazione/IRREDENTE/medaglie_infoibati.htm (Cnj sta per Coordinamento nazionale per la Jugoslavia), ultimo accesso 8 ottobre 2016. Qualche eco i due casi hanno avuto anche sulla stampa nazionale, anche a seguito di alcune interrogazioni parlamentari; si vedano per esempio "Corriere della sera", 23 marzo e 25 aprile 2015; "Il Fatto quotidiano", 25 aprile 2015.
} 
za" dei governi democratici e antifascisti del secondo dopoguerra l'accettazione del "diktat" del trattato di pace e il conseguente esodo, obnubilando le responsabilità del fascismo nell'aver condotto l'Italia in guerra a fianco dei nazisti e, sempre a fianco dei nazisti, nell'aver aggredito e smembrato la Jugoslavia, che al tavolo della pace poteva quindi presentarsi tra le fila delle vittime e dei vincitori. Ancora peggio, la vicinanza fra il Giorno del ricordo e la Giornata della memoria della Shoah, celebrata il 27 gennaio, sembrava fatta apposta per suscitare fraintendimenti e confusione, che puntualmente si sono verificati, anche se in misura ridotta. Del resto, tale appunto era l'intendimento esplicito di alcune frange più radicali dell'associazionismo degli esuli, contigue all'estrema destra italiana, da tempo impegnate a presentare foibe ed esodo quali atti genocidari, bisognosi di un riconoscimento pari a quello della Shoah ${ }^{25}$. Quel che lascia più perplessi, al riguardo, è non solo che una certa condiscendenza verso un'impostazione del genere fosse silenziosamente presente anche nelle associazioni più moderate della diaspora giuliano-dalmata, ma che il legislatore, pur avvertito, abbia preferito non accorgersene.

Tuttavia, la debolezza culturale della destra italiana, soprattutto in campo storico, ha procurato serie difficoltà ai suoi sostenitori tutte le volte in cui si è trattato di passare dalla retorica celebrativa alla riflessione storiografica, alla divulgazione di alto profilo e — soprattutto - alla costruzione di percorsi didattici. Su questo versante quindi, il ruolo più incisivo è stato svolto, dopo qualche iniziale perplessità, da un'agenzia di ben diverso orientamento, vale a dire la rete degli Istituti per la storia della resistenza, coordinati dall'Istituto nazionale per la storia del movimento di liberazione in Italia. In questo modo, è stato possibile allargare lo sguardo a quelle che la legge istitutiva del Giorno del ricordo chiama sbrigativamente "le altre vicende" del confine orientale, recuperando una dimensione di lungo periodo e problematizzando la funzione assunta in quegli eventi dagli italiani, che non sempre ne furono le vittime, come ben risulta quando si parli di "fascismo di confine" ovvero delle occupazioni italiane nei Balcani durante la seconda guerra mondiale. Com'è piuttosto ovvio, il Rapporto finale della Commissione italo-slovena ha costituito uno dei principali riferimenti per le iniziative delle rete degli Istituti della resistenza, mentre è stato invece completamente trascurato nelle attività promosse dai gruppi nazionalisti, che all'atto della pubblicazione del testo non avevano mancato di accusare i suoi estensori di alto tradimento, con tanto di denunce alla magistratura ed esposti alle autorità accademiche.

${ }^{25}$ F. Focardi, Rielaborare il passato, cit., p. 268; Damiano Garofalo, La memorializzazione delle Foibe e il paradigma della Shoah. Storia, politica, televisione, "Officina della Storia", numero monografico Media e storia, 2015, n. 14. Più in generale, sulla narrazione pubblica delle foibe vedi Federico Tenca Montini, Fenomenologia di un martirologio mediatico. Le foibe nella rappresentazione pubblica dagli anni Novanta ad oggi, Udine, Kappa Vu, 2014. 


\section{Un bilancio}

Rimanendo dunque all'interno di una prospettiva esclusivamente italiana, il bilancio del Giorno del ricordo a un decennio dalla sua istituzione presenta luci e ombre.

Sul piano comunicativo, vanno ricordati i non pochi beceri esempi di uso pubblico della storia, sostenuti anche da iniziative mediatiche di dubbio gusto. Al riguardo, la palma in negativo va certo assegnata alla fiction Il cuore nel pozzo dedicata alle foibe istriane dell'autunno 1943, prodotta nel 2005 dalla Rai: un vero coacervo di luoghi comuni, guardando il quale lo spettatore assai poco poteva capire di quanto accaduto, e quel poco era pure sbagliato. Con pervicacia degna di miglior causa, la Rai continua a infliggerlo agli spettatori ogni 10 febbraio, moltiplicando i danni. Assai diverso per sensibilità e riscontri risulta invece il musical Magazzino 18, proposto nel 2013 da Simone Cristicchi, che si segnala per aver suscitato larghi consensi trasversali alle appartenenze nazionali e politiche. Prodotto dal Teatro stabile del Friuli-Venezia Giulia, lo spettacolo ha incontrato un successo clamoroso, riuscendo a dar voce a diverse fra le memorie dolenti di confine, ponendole fra loro in dialogo e non in contrapposizione. Al di là di ogni giudizio sul valore artistico dello spettacolo e sul rispetto della storia da parte di un prodotto artistico, il punto è che il sostegno offerto a Magazzino 18 da parte di quasi tutte le forze politiche e le istituzioni triestine non costituisce una scelta estemporanea, ma si inserisce invece all'interno di un percorso di riconciliazione avviato da qualche anno fra le organizzazioni dei profughi giuliano-dalmati e quelle della minoranza slovena in Italia. Tappa importante di tale percorso è stato nel 2009 un pubblico dibattito promosso a Trieste dai quotidiani "Il Piccolo" e "Primorski Dnevnik", i cui protagonisti sono stati il presidente dell'Associazione nazionale Venezia Giulia e Dalmazia Lucio Toht, zaratino di origine ungherese, e il senatore Miloš Budin, esponente della comunità slovena di area ex comunista. Qualche anno dopo, Budin avrebbe presieduto il Teatro stabile e l'Anvgd avrebbe fortemente sostenuto il musical, che ha poi iniziato a girare l'Italia, mentre a Simone Cristicchi nel 2016, ovviamente in occasione del Giorno del ricordo, il Comune di Trieste ha concesso la cittadinanza onoraria ${ }^{26}$. In questa occasione quindi, l'operazione mediatica non ha avuto né obiettivi né effetti divisivi, ma conciliativi, il che fa una certa differenza con le precedenti esperienze. Ciò non toglie, evidentemente, che a qualcuno non siano piaciuti affatto né lo spettacolo, né la strategia politica di cui Magazzino 18 ha rappresentato un momento significativo ${ }^{27}$.

\footnotetext{
${ }^{26}$ La relativa mozione è stata approvata dal Consiglio comunale con tre voti contrari, mentre la successiva delibera è passata con una sola astensione.

${ }^{27}$ Critiche sono venute, con motivazioni antitetiche, dalle associazioni dei profughi di estrema destra e da alcuni gruppi di estrema sinistra, per i quali vedi i già cit. www.nuovaalabarda. org; www.cnj.it/documentazione/paginafoibe.htm; www.wumingfoundation.com/giap/.
} 
Sul versante della didattica, come spesso accade in occasione delle giornate memoriali, di fronte a un'inedita domanda di storia le risposte sono state disparate. Le più facili naturalmente sono state le lezioni ad hoc, affidate prevalentemente a esperti esterni provenienti dalle terre di confine, anche a seguito della generale carenza di competenze sull'argomento. In alcuni casi, la delega dell'intervento didattico a testimoni della stagione delle foibe e dell'esodo ha posto i problemi oramai ben noti di appiattimento della ricostruzione del passato sul vissuto di gruppo, irrigidito dalla rielaborazione collettiva e, nello specifico, adeguato ai canoni formulati dalle associazioni degli esuli giuliano-dalmati. In genere tuttavia, la compresenza di storici e testimoni ha consentito di bilanciare gli approcci. Nel corso del tempo infine, sono state sviluppate esperienze di grande interesse, che pongono la giornata del 10 febbraio come tappa di percorsi meditati di riflessione in classe e utilizzano le vicende dell'Adriatico orientale come chiave introduttiva alla storia delle contemporaneità europea. Lo stimolo in tale direzione è venuto da alcuni Istituti per la storia della resistenza e poi anche il ministero dell'Istruzione, dell'università e della ricerca ha avviato numerose iniziative di formazione degli insegnanti nell'ambito del Gruppo di lavoro per la conoscenza della storia degli esuli dell'Istria, di Fiume e della Dalmazia.

Sul piano della ricerca, il repentino e massiccio accendersi dell'interesse politico e mediatico per la storia dell'Adriatico orientale ha favorito in maniera cospicua sia gli studi sia la diffusione delle conoscenze sui rapporti fra l'Italia e gli slavi del Sud. Inoltre, ha consentito di far spazio, vuoi nel dibattito accademico vuoi a livello didattico, ad alcuni nodi non molto frequentati da parte degli storici italiani - come quelli relativi ai grovigli identitari e ai processi di nazionalizzazione parallela e competitiva in aree mistilingui, al trattamento delle minoranze da parte degli "stati per la nazione" novecenteschi, agli spostamenti forzati di popolazione - e ha offerto nuovi materiali e spunti comparativi alle ricerche in corso sui rapporti fra i partiti comunisti europei e sull'evoluzione delle forme della violenza politica durante e dopo le due guerre mondiali.

Naturalmente non sono mancate voci di dissenso nei confronti della celebrazione, anche se piuttosto circoscritte nello spazio pubblico ${ }^{28}$. Di prevalenza hanno denunciato le esagerazioni propagandistiche diffuse da associazioni nazionaliste e formazioni neofasciste, cui spesso i media attingono con abbondanza, anche con infortuni clamorosi, come quello delle foto di civili sloveni fucilati da soldati italiani scambiate per immagini di infoibati ${ }^{29}$. In alcuni ca-

\footnotetext{
${ }^{28}$ Vedi al riguardo i già citati www.nuovaalabarda.org; www.cnj.it/documentazione/paginafoibe.htm; www.wumingfoundation.com/giap/; www.diecifebbraio.info; terrerosse.altervista. org. Vedi anche Federico Tenca Montini, Confini stridenti. Nazionalismo antislavo e giorno del ricordo, "Zapruder", 2015, n. 36, pp. 126-132.

${ }^{29}$ L'immagine dei contadini fucilati a Dane, in Slovenia, il 31 luglio 1942, è girata a lungo sul web come rappresentazione dell'uccisione di civili italiani da parte jugoslava, nonostante le ripetute smentite e le esortazioni a maggior attenzione inviate per esempio dall'Irsmlfvg a tut-
} 
si però, il loro impegno di "controinformazione", fortemente connotato in senso ideologico e non privo di venature nostalgiche nei confronti del regime comunista jugoslavo, ha investito la sostanza stessa di processi storici fortemente traumatici e complessi - come quelli che hanno generato le stragi delle foibe e l'esodo - , riproponendo le versioni giustificatorie a suo tempo diffuse dal regime di Tito e scadendo nel negazionismo.

Ancor più articolato è il discorso qualora si allarghi lo sguardo oltre i confini italiani, per valutare l'impatto del Giorno del ricordo sul complesso dei rapporti fra l'Italia e gli stati ex jugoslavi. Qui infatti la logica che ha presieduto all'approvazione della legge 92/2004 appare chiaramente antitetica a quella che dieci anni prima aveva suggerito la costituzione delle due commissioni bilaterali, italo-slovena e italo-croata. L'intento delle commissioni - come abbiamo visto - era stato quello di evitare che la sovrabbondanza di memorie divise non elaborate criticamente finisse per colonizzare la storia in un tornante politico cruciale, con gravi danni per le relazioni fra stati e popoli contigui. L'obiettivo del Giorno del ricordo è stato invece proprio quello di recuperare una di tali memorie, quella degli italiani d'Istria, Fiume e Dalmazia, non a torto considerata a rischio di estinzione, e di attribuirle un ruolo significativo all'interno del processo di ricostruzione di un'identità nazionale italiana da più parti considerata eccessivamente "debole". Tale operazione ha indubbiamente raggiunto alcuni degli obiettivi che si era proposta: la storia dolente degli italiani provenienti dai territori passati alla Jugoslavia dopo il secondo conflitto mondiale è uscita dal cono d'ombra che l'aveva celata per decenni, così com'è avvenuto per altre vicende non troppo dissimili di tante altre popolazioni dell'Europa centro-orientale; i ricordi di vita di cui costoro erano portatori sono stati salvati grazie a massicce raccolte di testimonianze, che hanno dato luogo anche a opere di elevato valore scientifico; molti dei sopravvissuti alle violenze e agli esodi di allora, come pure i loro congiunti, sono stati posti nella condizione di sanare almeno in parte quelle ferite della memoria che i lunghi anni di amnesie private, collettive e pubbliche avevano lasciato suppurare.

È avvenuto però con il Giorno del ricordo quel che è accaduto anche ad altre fra le molte iniziative - di per sé per tanti versi meritorie - di recupero delle "memorie negate", fiorite in Europa negli ultimi anni, magari l'una in risposta alle altre ${ }^{30}$. Il loro presupposto comune è molto spesso infatti costituito dall'esigenza di riscoprire, irrobustire, valorizzare una specifica identità nazionale, senza tener adeguato conto di un fatto fondamentale: le tragedie che hanno pro-

ti gli Istituti della rete, ed è stata quindi utilizzata, anche inconsapevolmente, come locandina di iniziative legate al 10 febbraio, fino a venir proposta in Tv a Porta a Porta del 13 febbraio 2012, suscitando vibrate proteste.

${ }^{30}$ Vedi al riguardo le considerazioni svolte in Guido Crainz, Il difficile confronto fra le memorie divise, in Guido Crainz, Raoul Pupo, Silvia Salvatici (a cura di), Naufraghi della pace. Il 1945, i profughi e le memorie divise d'Europa, Roma, Donzelli, 2008, pp. 175-191. 
vocato le vittime che in quelle giornate memoriali vengono commemorate sono in buona parte legate proprio all'urto fra identità nazionali reciprocamente sopraffattrici. Ne segue che le memorie dolenti sono strutturalmente memorie di divisione. Appare assai chiara la contraddizione fra tale esito, in buona misura inevitabile, e le istanze invece non solo di collaborazione bilaterale ma di una progressiva integrazione continentale maturate nei medesimi anni. In altri casi europei, a temperare la contraddizione sono intervenute tempestivamente le istituzioni, con atti dall'elevato significato simbolico che non sempre hanno conseguito del tutto i risultati attesi, ma che perlomeno hanno indicato l'esistenza di una sensibilità politica piuttosto viva. Sulle sponde adriatiche invece, i tempi delle politiche conciliative sono stati più rilassati.

All'istituzione in Italia del Giorno del ricordo ha risposto a stretto giro l'istituzione in Slovenia di una Festa del ritorno del Litorale sloveno alla madrepatria da tenersi ogni 15 settembre, data dell'entrata in vigore nel 1947 del trattato di pace di Parigi che assegnava alla Jugoslavia la sovranità su buona parte del Litorale. Con chiaro intento polemico, le relative celebrazioni non si sono però svolte nel Goriziano o nel Postumiese, territori a popolamento storico sloveno entrati a far parte della Slovenia fino appunto dal 1947, bensì nei comuni costieri di Capodistria e Pirano, all'epoca a maggioranza italiana, poi svuotatisi con l'esodo, nel 1947 sottoposti da parte jugoslava a mera occupazione militare fiduciaria in attesa della costituzione del Territorio libero di Trieste, trasferiti di fatto alla Jugoslavia con il Memorandum di Londra del 1954 e de jure con il trattato di Osimo del 1975. La Croazia invece non ha assunto iniziative del genere, ma nel 2007 l'allora presidente della repubblica, Stipe Mesić, è stato protagonista di una dura polemica pubblica con il presidente italiano Giorgio Napolitano. Attorno alla data fatidica del Giorno del ricordo si sono infatti succedute alcune dichiarazioni dei vertici delle due repubbliche che rispolveravano un linguaggio che si credeva sepolto, almeno a quei livelli: per un verso, la definizione delle foibe come "una reazione ai crimini fascisti"; per l'altro, la denuncia di un "moto di odio e di furia sanguinaria e di un disegno annessionistico slavo che prevalse nel Trattato di pace del 1947 e che assunse i sinistri contorni di una pulizia etnica". Forti critiche alle parole di Napolitano, se pur soltanto in via riservata, sono venute anche dal presidente della Repubblica slovena, Drnovšek, che alle parole del capo dello Stato italiano ha contrapposto proprio i contenuti del rapporto finale della Commissione italo-slovena.

Non è difficile notare, dunque, quanto le esigenze di politica interna avessero innescato una deriva che si collocava agli antipodi rispetto allo spirito delle commissioni bilaterali e che rischiava di procurare gravi danni alle relazioni fra Italia, Slovenia e Croazia. Di fronte al conclamato incidente internazionale, di tale pericolo si sono rese conto anche le diplomazie dei tre paesi, che hanno invertito la rotta. Ne è seguito un complesso negoziato, alla fine del quale si è arrivati al grande gesto simbolico di riconciliazione. Il 20 luglio 2010 i presidenti delle repubbliche di Italia, Slovenia e Croazia si sono ritrovati assieme 
a Trieste per rendere comune omaggio ad alcuni dei luoghi memoriali dei conflitti novecenteschi, come la sede dell'ex Narodni Dom - il cui incendio giusto novant'anni prima aveva segnato l'inizio delle persecuzioni fasciste contro gli sloveni e i croati trovatisi da vivere dopo il 1918 nel regno d'Italia - e il monumento che ricorda l'esodo degli italiani da Zara, a Fiume e dall'Istria dopo la seconda guerra mondiale. La giornata riconciliatoria è stata poi conclusa da un grande concerto per la pace, diretto da Riccardo Muti nella principale piazza cittadina affacciata sul mare, già scenario di infinite manifestazioni patriottiche e politiche, campo di battaglia preferito tra militanti di opposte tendenze e teatro di sanguinose repressioni poliziesche. A sottolineare la rinnovata volontà di disinnescare le mine del passato, l'anno successivo i presidenti di Italia e Croazia si sono ritrovati a Pola, per assistere a un nuovo concerto della pace, questa volta nella suggestiva sede dell'arena romana.

Dunque, un lieto fine? ${ }^{31}$ Forse, perché la storia va sempre avanti, ma alcune delle maggiori criticità registratesi in un recente passato sembrano comunque dietro le spalle. Certamente, il ruolo delle commissioni storiche non può venir paragonato a quello delle "commissioni di riconciliazione", perché un'impresa del genere va condotta contemporaneamente sul piano della storia - in quanto disciplina critica - , della memoria e della politica: e in effetti, anche se in maniera tutt'altro che lineare, un processo del genere è stato realmente avviato, a molti livelli, fra i quali quello della ricerca storica è forse il più semplice. Ben più complessa ad esempio è la gestione delle memorie, dove a venir messi in gioco sono i vissuti e i depositi identitari. Al riguardo, certo non hanno giovato gli equivoci generati nel corso degli anni Novanta dall'introduzione nel dibattito culturale e politico italiano della categoria di "memoria condivisa". Più recentemente, ma la prudenza è d'obbligo, sembra che quell'infelice formula stia cedendo terreno rispetto ad altre concettualizzazioni, come il "rispetto delle memorie" - che, in quanto soggettive, non sono interscambiabili - e la "purificazione delle memorie" - un'espressione quest'ultima che, pur risultando di ovvia derivazione religiosa, implica semplicemente la consapevolezza che anche le memorie delle comunità cui si sceglie di aderire presentano zone oscure, che richiedono di problematizzare le autorappresentazioni esclusivamente vittimistiche e di aprirsi all'ascolto delle memorie degli altri. Nel caso specifico dell'Adriatico orientale, non mancano fortunatamente le buone pratiche. Fra queste rientra senza dubbio il percorso riconciliativo - di cui si è già detto a proposito di Magazzino 18 - che ha visto per protagoniste le maggiori organizzazioni degli esuli giuliano-dalmati, della minoranza slovena in Italia

${ }^{31}$ Di ciò, come pure del valore positivo del Giorno del ricordo, sembrano convinti sei fra i sottosegretari di vari governi italiani (Roberto Antonione, Miloš Budin, Piero Fassino, Alfredo Mantica, Roberto Menia, Umberto Ranieri) riunitisi a Trieste il 29 febbraio 2016, nonostante il neo nella distensione con Slovenia e Croazia rappresentato dall'irrisolto problema del risarcimento dei beni abbandonati dagli esuli; vedi al riguardo "Il Piccolo", 1 marzo 2016. 
e di quella italiana in Slovenia e Croazia; tanto più che è stata tale rete a svolgere un ruolo attivo nella "ricucitura" diplomatica che ha condotto all'incontro dei presidenti delle tre repubbliche. Altri due esempi val la pena di ricordare: il primo è rappresentato dalla costituzione a Trieste di un Laboratorio della memoria, impegnato dal 2012 in una vasta gamma di attività di studio e comunicazione di taglio fortemente internazionale sul rapporto storia-memoria ${ }^{32}$; il secondo, dalla fondazione a Nova Gorica dell'associazione ecclesiale Concordia et pax, promotrice nell'ambito delle diocesi situate dalle due parti del confine italo-sloveno di numerose iniziative di riconciliazione dal forte valore simbolico, quali i pellegrinaggi ai campi di concentramento per civili jugoslavi in Italia (come Gonars), alla caserma Piave di Pamanova - luogo di tortura fascista - e ai campi per prigionieri italiani nella ex Jugoslavia (come Borovnica) ${ }^{33}$.

Per converso, rimangono ancora alcuni cultori delle memorie antagoniste, lungo discriminanti nazionali o ideologiche, com'è del resto legittimo che sia; ma, all'interno di un contesto generale pacificato e di progressivo superamento dei confini, la loro incidenza risulta limitata a circuiti specifici ed allo spazio virtuale $^{34}$. Il problema è che i confini ritornano: a premere per la loro rimaterializzazione sono le nuove spinte impresse dal quel flusso di migranti che, pur essendo tutt'altro che imprevisto, ha colto egualmente impreparate le istituzioni sia degli stati nazionali sia comunitarie, determinando una condizione crescente di crisi. Nelle crisi, si sa, può accadere di tutto, anche che si riattivino faglie dormienti. Un esempio, fortunatamente solo curioso, è dato dal riemergere, negli anni della crisi finanziaria italiana, di un movimento a favore del Territorio libero di Trieste, avente come parola d'ordine quella di non pagare le tasse italiane e come icona quella di Vladimir Putin: un fenomeno tutto da ridere, se non si fosse dimostrato capace di ottenere sostegni più o meno palesi da ambienti di estrazione nazionale e politica assai diversa e di suscitare pugnaci entusiasmi fra lavoratori portuali e ultras del $\mathrm{calcio}^{35}$.

Naturalmente, a risolvere i problemi del futuro non possono essere gli studiosi del passato, ma chi opera politicamente nel presente. Tuttavia, la densità di quanto è accaduto ma forse non ancora del tutto metabolizzato, suggerisce di

32 Il Laboratorio è stato costituito dall'amministrazione provinciale assieme al Comune di Trieste, all'Università degli studi di Trieste, all'Irsml, alle sezioni locali dell'Anpi-Vzpi, all'Aned, all'Anppia, all'Avl, alla Sissa, all'Irci, alla Federazione delle Associazioni degli esuli istriani, fiumani, dalmati e all'Anvgd.

${ }^{33}$ Per un quadro delle iniziative si veda all'indirizzo web www.concordiaetpax.org/, nonché Franco Miccoli, Sentieri di memoria e di riconciliazione, "Nuova iniziativa isontina", 2014, n. 66, pp. 31-34.

${ }^{34} \mathrm{Si}$ veda, per un verso, all'indirizzo web www.unioneistriani.it/; per l'altro, i siti già citati alla nota 28.

${ }^{35}$ Per le informazioni di fondo e alcuni primi spunti analitici si veda agli indirizzi web www.limesonline.com/se-trieste-rinnega-litalia/57282; www.balcanicaucaso.org/aree/Italia/Territorio-Libero-di-Trieste-141026; www.wumingfoundation.com/giap/?p=14409, ultimi accessi 10 ottobre 2016. 
non rimuovere la questione della responsabilità degli storici. È, questo, un tema assolutamente desueto nel dibattito culturale italiano, per ragioni che non è qui il caso di indagare. Chi però si è misurato con la storia di frontiera sa bene che il problema non può venire eluso: non perché dalla storia sia realmente possibile trarre gran copia di ammaestramenti, ma perché il passato costituisce un deposito ricchissimo di argomenti e pretesti da utilizzare per alimentare conflitti. È successo e continua a succedere. Per questo, alcune generazioni di storici di frontiera hanno posto come loro obiettivo, pur nella diversità delle formazioni culturali, un impegno serrato contro i pregiudizi, le banalizzazioni, le verità di comodo, gli schematismi, le comparazioni indebite, contrapponendovi la ricostruzione critica dei processi storici, il loro puntuale riferimento ai diversi contesti, l'articolazione delle reti causali. È una combinazione di metodo rigoroso e di passione civile che può venir ancora messa in gioco per aiutare a comprendere lo spessore problematico delle nuove emergenze.

Ultimo tassello, quello della pietas. Anch'essa è dimensione poco frequentata nella ricerca storica, e a ragione, perché nella fase analitica freddezza e distacco costituiscono i requisiti indispensabili per un approccio critico verso oggetti storici incandescenti. Ma ciò non significa indifferenza: dietro i meccanismi, le strutture, le appartenenze, ci sono sempre gli esseri umani. Può capitare allora di osservare, per esempio, che nelle rovine di un centro di raccolta profughi in cui migliaia di esuli istriani trovarono un asilo tanto precario che alcuni bambini vi morirono di freddo, oggi cercano riparo altri esuli, cacciati da vicende ancor più sanguinose e almeno altrettanto bisognosi di accoglienza ${ }^{36}$. Cercar di capire e provare a spiegare, anche mettendo in campo tutti gli strumenti della conoscenza storica, diventa qui un fatto di coscienza.

${ }^{36}$ Testimonianza di Fiore Filippaz sulla morte della sorella Marinella, più volte resa agli organi di stampa, da ultimo nella trasmissione speciale per il 10 febbraio 2016 della sede Rai del Friuli-Venezia Giulia. 